\title{
Surface-enhanced Raman scattering sensors for biomedical and molecular detection applications in space
}

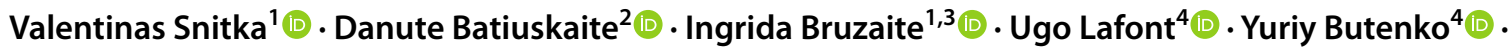 \\ Christopher Semprimoschnig ${ }^{4}$
}

Received: 30 October 2020 / Revised: 10 February 2021 / Accepted: 17 February 2021 / Published online: 8 March 2021

(c) CEAS 2021

\begin{abstract}
The detection of molecular traces in the environment is a technical problem that is critical in pollutant control procedures at all stages of spacecraft assembly, in space flight, as well as in other technological processes such as food production, medical diagnostics, environmental control, warfare. However, in the aerospace industry, it is necessary to detect molecular traces of contaminants with extreme sensitivity, as even concentrations as low as part-per-billion (ppb) can be critical during long missions. The high sensitivity of the Volatile Organic Compounds (VOCs) detection within the air can be a challenge because of the poor affinity of VOC's to the metal surface of the sensor substrate. In this work, we present a surface-enhanced Raman scattering (SERS) spectroscopy technique as a highly sensitive and selective molecular sensor for gas trace detection not sensitive to molecules adsorbtion on sensing element. The developed hybrid SERS platform for molecular trace detection is supported by the hybrid nanoplasmonic porous silicon membrane in conjunction with micropump to achieve the trace level detection of VOCs in the environment. The combination of silicon membrane, made by electrochemical etching of the microchannels in the silicon chip, with chemical deposition of the silver nanoparticles inside the channels, produce a porous Ag nanoparticles membrane with a high density of plasmonic nanostructures ("hot spots"). The micropump integrated with the SERS sensor, pump the air with VOC's molecules through the plasmonic membrane "hot spots" to increase the probability of interaction of VOC's molecules with SERS substrate and to increase the enhancement factor. The sensor chip structure was designed, gas flow in the sensor was simulated, and the sensor was fabricated using 3D printing. The limit of detection of hydrazine with concentration level $10^{-12} \mathrm{M}$ from solution and the vapor phase $0.1 \mathrm{ppm}$ was demonstrated. The anisole vapors with concentration $0.5 \mathrm{ppb}$ spectra in the air were recorded. Our results demonstrate that plasmonic membrane can be used as a high enhancement factor SERS sensor for many pollutants molecules detection with the nanomolar sensitivity and can be applied in the design of sensors for space applications, environment control, biomedical diagnostic.
\end{abstract}

Keywords Sensing $\cdot$ Volatile compounds $\cdot$ Surface-enhanced Raman scattering

\section{Introduction}

The detection of molecular traces in the environment is a technical problem that is critical in pollutant control procedures at all stages of spacecraft assembly, in space flight, as well as in other technological processes such as food production, medical diagnostics, environmental control, warfare. The growing technical requirements for the cleanliness of spacecraft, their equipment, and the instruments used are a consequence of the growing sensitivity of the equipment

Valentinas Snitka

vsnitka@ktu.lt; vsnitka@gmail.com

Extended author information available on the last page of the article used and the longer duration of flight missions[1]. Several studies have identified the most important and critical pollutants occurring in space facilities and compiled a list of them $[2,3]$, and monitoring methods have been developed $[4,5]$. However, the need for methods for measuring the molecular traces of contaminants and biological hazards with higher sensitivity than those currently in use remains an issue [6]. The technical challenges associated with trace contaminant control engineering during the ISS's early assembly stages have been presented and discussed in several works and the engineering analysis techniques and tools are directly applicable to future crewed space exploration missions and programs [7, 8].To adequately design a contamination control systems an active contamination control design options must 
be thoroughly reviewed and understood. Future space missions to other planets will require stringent cleanliness control of all onboard instruments to avoid false identifications of organic molecules possibly originating from spacecraft. Equally, rigorous cleanliness control is crucial for analyses of space return samples [9]. Monitoring the quality of the spacecraft environment is required during human space flight to maintain the health of crew members and vehicle systems. Environmental quality accounts for contaminants found in air and water, microbial growth, acoustics, and radiation exposure. The role of polycyclic aromatic hydrocarbons, bacteria, and amino acids will be paramount for future exploration missions featuring an astrobiological component [10]. Some major atmospheric constituents $\left(\mathrm{O}_{2}\right.$ and $\left.\mathrm{CO}_{2}\right)$ have been monitored in real-time for decades, but the majority of environmental monitoring has relied on archival sampling techniques, in which samples are collected from the spacecraft and returned to Earth for analysis. While environmental assessment from archival samples has been adequate for short missions, such as those on the Space Shuttle, longer missions aboard the International Space Station (ISS), in which sample return may surpass 6 months after collection, have emphasized the need for more extensive real-time environmental monitoring. This fact is especially true as ESA and other space agencies move toward more distant exploration missions when there will be limited ground support and no opportunity for archival sample return. Major constituents in the ISS atmosphere $\left(\mathrm{O}_{2}, \mathrm{CO}_{2}, \mathrm{~N}_{2}, \mathrm{H}_{2}\right.$, and $\left.\mathrm{CH}_{4}\right)$ are monitored by the Major Constituent Analyzer (MCA), a magnetic sector mass spectrometry-based instrument. The Air Quality Monitor (AQM), based on gas chromatography, measures compound-specific concentrations of a targeted list of volatile organic compounds (VOCs) selected based on their potential toxicological effects on crew health or detrimental effects on specific space apparatus systems [11]. Water quality is monitored in real-time by the Total Organic Carbon Analyzer (TOCA) and the Colorimetric Water Quality Monitoring Kit. The TOCA measures the total organic carbon (TOC) load in the ISS water systems but does not provide compound-specific information. While the TOCA provides excellent trending data regarding total organics and the overall water quality on the ISS, the inability to determine which compounds are responsible for any TOC increases makes it difficult to implement effective mitigation plans. Events beginning in June 2010 highlight the need for specific chemical information about the presence of organic compounds in ISS water [12]. The use of the novel chemical compounds detecting and identification techniques for both air and water monitoring would provide improved information over existing systems while also fulfilling the needs of future exploration missions by reducing the amount of hardware needed for environmental monitoring. There are currently several competing technologies and devices on the market for the detection of chemical compounds in the gas phases [13]. All of these devices do not provide fully optimal characteristics, and differences in cost, sensitivity, measurement speed, specificity, accuracy, and complex determination of the properties of the molecules being measured are a major challenge. In this context, the nanotechnology-based sensing techniques, namely the nanoplasmonic based surface-enhanced Raman scattering (SERS) spectroscopy, and application of novel nanomaterials for sensing substrates can be a promising way not only to improve the AQM and TOCA systems but to be applied for the detection of organic molecules on other planets and space objects (meteorites, comets) because of its ability to detect a single molecule [14]. The new hybrid porous materials $[14,15]$ with ultrahigh molecular absorbing capacities (graphene, silica aerogel, foams) are moving the SERS technique into new areas of application, including COVID-19 virus diagnostics. Because SERS is more sensitive, cheaper, faster, and easier to perform than other assays based on fluorescence or the reverse transcriptase-polymerase chain reaction, it could prove ideal for detecting and studying viruses in the future [16-18]. Novel miniaturized Raman spectroscopy systems, combined with SERS spectroscopy open new horizons in molecular and biosensing, leading to picomolar concentrations sensitivity level, low cost, and weight, possibility to get chemical and biological information concerning the analytes $[19,20]$. SERS sensors can be an alternative to currently used methods, since they do not require special instrumentation, and do not need sample clean-up and derivatization procedures, thus being in general less-time consuming. Besides, an important advantage of this technique is that it may be used for in situ analysis. In this paper, we introduce the novel concept and results of experimental realization of space relevant molecules detection platform, based on the porous gas transparent membrane with a high density of localized surface plasmonic structures ("hot spots") for surface-enhanced Raman scattering (SERS) spectroscopy, combined with the environmental gas pumping system.

\subsection{Principle of operation of the surface enhanced Raman scattering measurement}

Some metal nanoparticles exhibit local surface plasmon resonance based on collective oscillations of their conduction electrons and it is the interaction of this effect with molecules that is the origin of plasmon-enhanced spectroscopy. The amplified re-emitted dipole fields excite the adsorbent, and if the resulting molecular radiation remains near resonance with the amplifying object, the scattered radiation will again be amplified. If the plasmonic structures are well designed, the field enhancement will scale as $\mathrm{E}^{4}$, where $\mathrm{E}$ local optical field. SERS system essentially brings together microscopy and spectroscopy technologies. A schematic of 
the setup used in our work is shown in Fig. 1. It consists of two main subsystems - an optical confocal microscope and a Raman spectroscopy system.

There are several key challenges when attempting to achieve a one molecule SERS sensitivity. These include:

- the quality of SERS substrate - its shape, metal type, size, and cleanliness;

- heating effects (power and the wavelength of the excitation beam);

- adhesion of the gas molecules on SERS surface;

- the temperature of the gas environment.

Generally, the low laser power regime is used when collecting SERS spectra from a sample consisting of a few molecules. The scanning mode can be used to avoid the destruction of analyte molecules by the excitation laser beam. Using the high-quality SERS substrate the enhancement of $10^{4} \mathrm{can}$ be achieved.

\section{Experimental methods}

\subsection{Fabrication of SERS substrates}

\subsubsection{Materials}

Hydrofluoric acid (48 wt.\% in $\mathrm{H}_{2} \mathrm{O}, \geq 99.99 \%$ ), $\mathrm{AgNO}_{3}$ ( $\geq 99.0 \%$ ), water (molecular biology reagent), ethanol ( $\geq 99.8 \%)$, methanol ( $\geq 99.9 \%)$, Crystal Violet (CV)

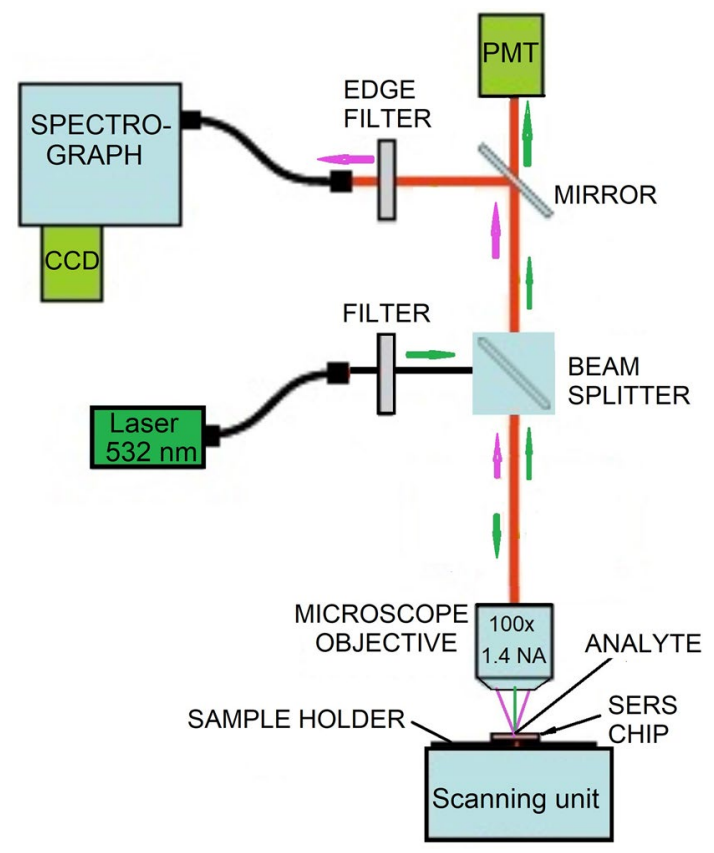

Fig. 1 Schematic of the setup for the acquisition of SERS spectra
( $\geq 95.0 \%)$, anisole ( $\geq 99.9 \%)$, hydrazine ( $\geq 98 \%)$, silicon wafers were purchased from Sigma Aldrich (www. sigmaaldrich.com/european-export.html). The silicon membranes with pores were purchased from SmartMembranes GmbH, Germany. All the reagents were used as purchased without further purification.

\subsubsection{Synthesis of 3D Ag/Si SERS substrates}

Silicon wafers [111] were cut into small pieces $(1.5 \times 1.5 \mathrm{~cm})$. The silicon wafers were abraded $(2 \mathrm{~min})$ with the silicon carbide paper P1500 grit to rough up the silicon surface. Such prepared silicon was washed with pure ethanol and dried under the nitrogen flow. Such prepared silicon wafers were kept in the closed Petri dishes until use. The synthesis of silver SERS substrates was based on direct silver ions reduction by elemental silicon. The silver precursor solution was prepared by dissolving $\mathrm{AgNO}_{3}$ in water to the final concentration of $10 \mathrm{mM}$. The prepared HF (1\%) and $\mathrm{AgNO}_{3}(10 \mathrm{mM})$ solutions were mixed in a ratio of $1: 1$ $\mathrm{v} / \mathrm{v}$. The 3D structured silicon wafers were immersed in the reaction mixture for the $20 \mathrm{~s}$ then immediately transferred to the pure water and finally dried using the nitrogen flow.

\subsubsection{Fabrication of Ag/silicon membrane SERS substrate}

Silicon membranes are formed from p-type silicon monocrystal wafers with a thickness of $200 \mu \mathrm{m}$. The micro-pores are etched through a silicon wafer using a photoelectrochemical process. The arrangement of micropores is formed by photolithography. Porous membranes with the size of $10 \times 10 \mathrm{~mm}$, pore diameter $6 \mu \mathrm{m}$, the distance between the pores $4 \mu \mathrm{m}$ were used for the production of SERS samples. The membranes used are gas permeable through the formed pores.

Before Ag precipitation, the membranes were cleaned with acetone and isopropanol to remove potential contaminants from the surface. The membranes were then activated by immersion in $8 \% \mathrm{HF}$ solution for $10 \mathrm{~s}$, washed in Milli-Q water, dried in a stream of $\mathrm{N}_{2}$, and immediately.

the drop of silver electroless deposition solution was put on the membrane surface. The solution of silver precursor for electroless deposition on the surface of the porous membrane was used as described in 3.1.2. The membranes with the drop were positioned horizontally for $1 \mathrm{~min}$ at room temperature on the surface of the high-grade polished cover glass. As a result, the silver solution penetrates the membrane channels, and reacting with silicon walls of the micropores fills the channel with the silver nanoparticles. The silver nanoparticle covered membrane-type SERS substrates were immersed in Milli-Q water to remove residual solution and finally dried using the $\mathrm{N}_{2}$ gas flow. 


\subsubsection{Characterization of SERS substrate}

The structure studies of SERS samples were performed using a scanning electron microscope (SEM) (Hitachi, S-3400 N). The efficiency of the SERS sensor plasmon field interaction with analyte molecules was investigated using CV dye monolayer and anisole vapor molecules. The $\mathrm{CV}$ deposition methodology used was analogous to that described in the previous work [21]. The formation of CV monolayer on $\mathrm{Ag} / \mathrm{Si}$ membrane was done in a water solution of $\mathrm{CV}\left(10 \times 10^{-9} \mathrm{M}\right)$ by immersion of the membrane into a solution for $2 \mathrm{~h}$. Finally, the substrate was washed in methanol to clean of unadsorbed molecules. The anisole vapor was prepared by the drop of anisole evaporated in a closed container with the SERS substrate. For the membrane SERS the anisole gas molecules were prepared by $\mathrm{N}_{2}$ bubbling through liquid anisole in a closed vessel connected with the gas inlet of the SERS measurement device.

\subsubsection{Operating principle of SERS membrane sensor}

The dynamic of gas flow through the membrane was investigated by a computer simulation experiment. To optimize the structure of the device the computer simulation using a finite element method (FEM) with the SolidWorks 3D CAD software was performed. The Flow simulation module (CFD) was used for the analysis of gas flow through the sensor chip to optimize the topology.

The task of topology design is to find a condition to produce on the SERS chip membrane output surface the gas flow vortexes and turbulence. The turbulent flow produces a higher rate of gas molecules interaction with plasmonic nanostructures ("hot spots") and higher sensitivity of the sensor.
A schematic of SERS membrane sensor structure is shown in Fig. 2. The simulation results can be found in the Supplement video.

\subsubsection{The 3D membrane device manufacturing}

The 3D plastic printing based on fused deposition manufacturing processes was selected for making the geometry of the SERS sensor. The material used for 3D printing was the acrylonitrile butadiene styrene (ABS, chemical formula $\left(\mathrm{C}_{8} \mathrm{H}_{8}\right) \mathrm{x} \cdot\left(\mathrm{C}_{4} \mathrm{H}_{6}\right) \mathrm{y} \cdot\left(\mathrm{C}_{3} \mathrm{H}_{3} \mathrm{~N}\right) \mathrm{z}$ plastic filaments. The printing was done layer by layer with a layer thickness of $0.1 \mu \mathrm{m}$ to ensure the high accuracy of the sensor structure. The Ultimaker Cura-4.1.0 pre-processor was used to generate G-code for printing control and convert AUTOCAD 3D model into G-code instructions for the 3D printer.

The printing process settings: extrusion temperature 245 ${ }^{\circ} \mathrm{C}$, build plate temperature $90^{\circ} \mathrm{C}$, the object infill $40 \%$; the layer height $0.1 \mathrm{~mm}$; print speed $60 \mathrm{~mm} \mathrm{~s}^{-1}$; plastic filament diameter $1.75 \mathrm{~mm}$. The design of the SERS membrane sensor is presented in Fig. 3.

\subsubsection{Gas pumping}

The commercial mp6-gas micropump from Bartels-microtechnik $\mathrm{GmbH}$ was used. The gas flow rate of $12 \mathrm{ml} \mathrm{min}^{-1}$ was selected. mp6-QuadEVA software and control board EVA was controlling the micropump (Fig. 4).

\subsubsection{Raman/SERS measurements setup}

Raman scattering spectra were measured at room temperature by back-scattering geometry with a Raman spectrometer (NTEGRA Spectra, NT-MDT Inc.). The calibration of

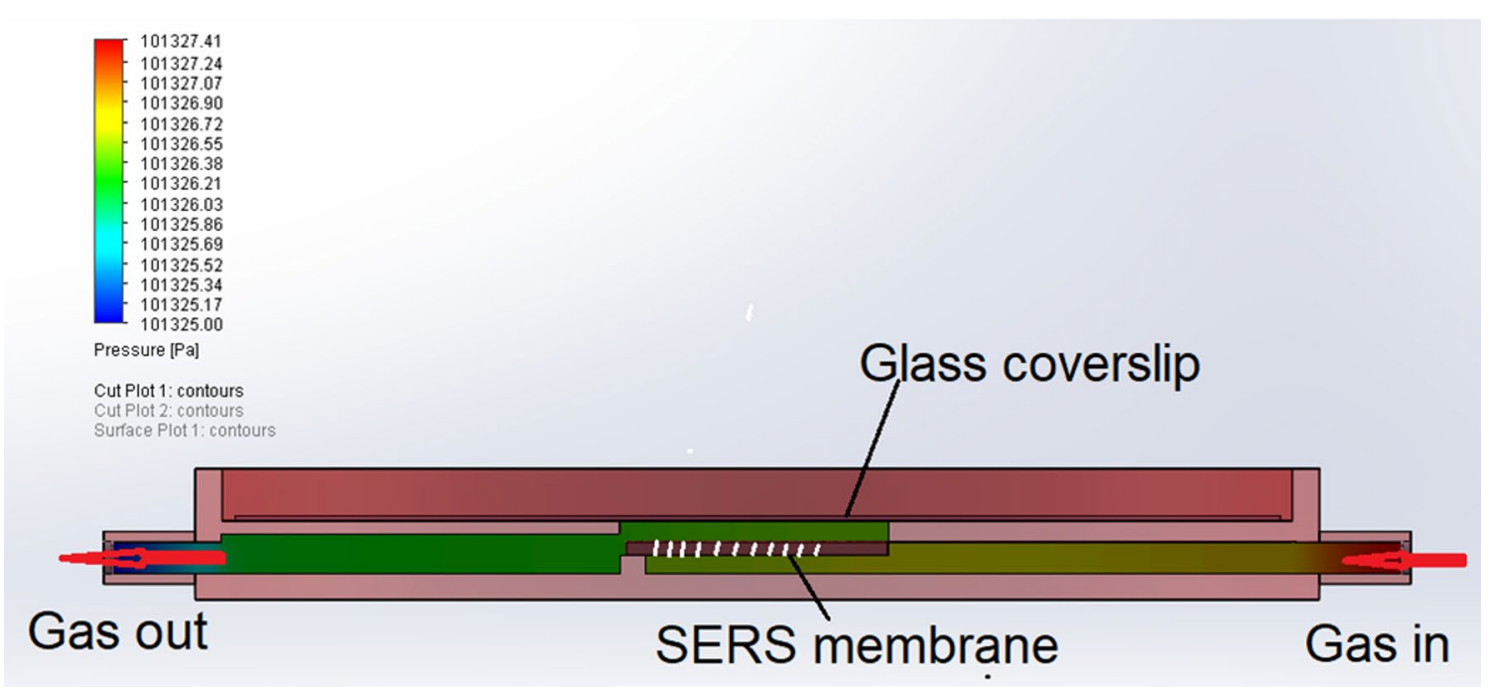

Fig. 2 Structure of membrane sensor for gas flow simulation 


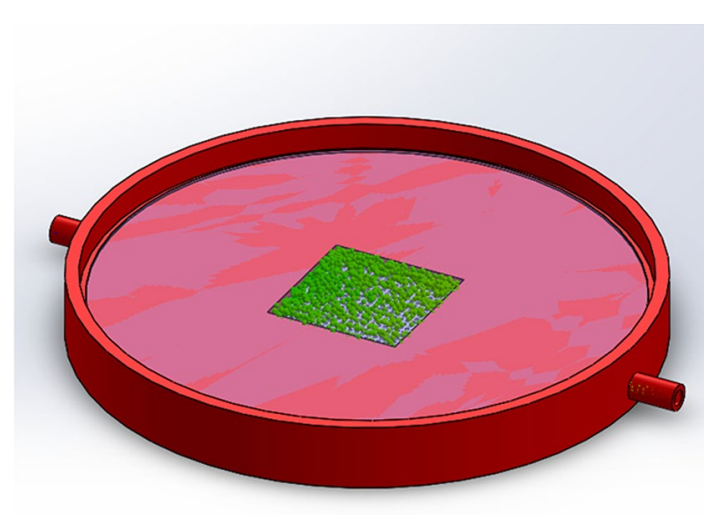

Fig. 3 Computer 3D model of SERS membrane sensor

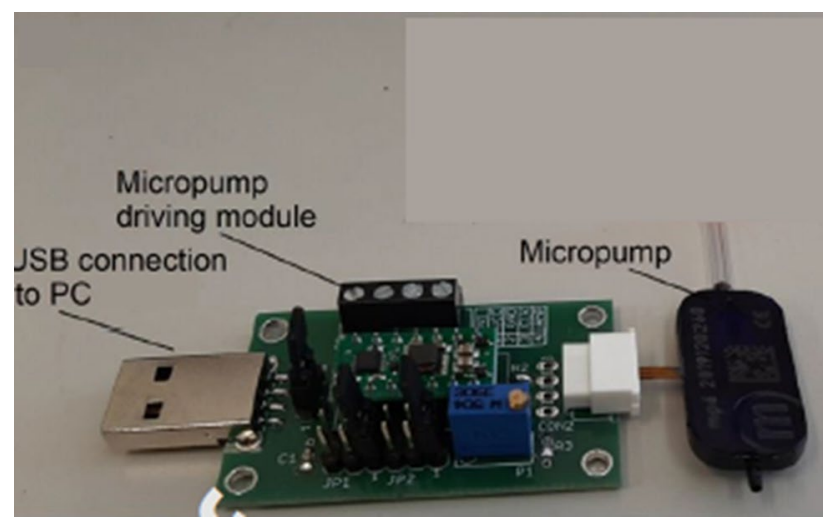

Fig. 4 Micropump used for SERS membrane gas sensor test

the instrument was done using a silicon LO phonon peak at $520 \mathrm{~cm}^{-1}$ as a reference point.

The excitation laser $532 \mathrm{~nm}$ line and a $100 \times$ objective (NA 1.49) were used. The laser beam focus spot diameter: $500 \mathrm{~nm}$. The excitation source optical beam power: $25 \mathrm{~mW}$ and the optical beam power on the sample: $1.7 \mathrm{~mW}$. The Raman scattering signal was detected by a thermoelectrically cooled $\left(-60{ }^{\circ} \mathrm{C}\right)$ charge-coupled device (CCD). The spectral resolution of the used grating was $1.1 \mathrm{~cm}^{-1}$. The Spectragryph 1.2.10 software for the pre-processing of the spectral data was used [22]. The schematic of the measurement setup is presented in Fig. 5.

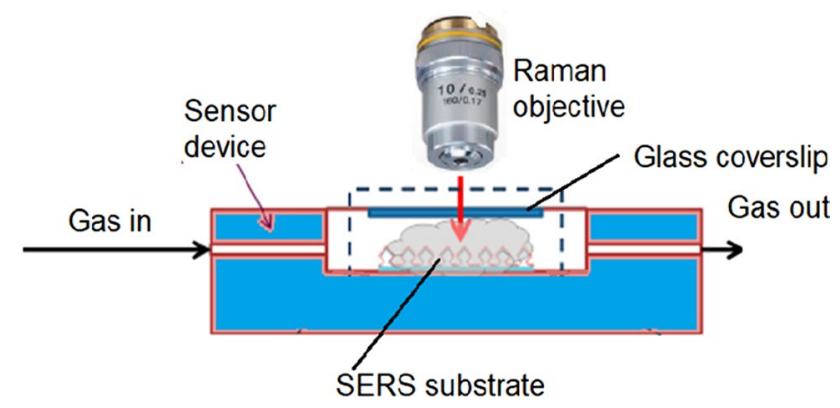

Fig. 5 Experimental setup for continuous SERS measurements in the gas phase

\section{Results and discussion}

\subsection{Characterisation of SERS substrates}

\subsubsection{Morphological characterization of the 3D Ag/Si SERS active substrates}

The proposed method allows the formation of the ripplestype 3D silver SERS substrates (Fig. 5) in an easy and cheap way. Such a type of silver SERS substrate has good repeatability in the structure as is shown in Fig. 6a. The relative enhancement of the Raman signal for R6G monolayer was found to be $10^{7}$ (Fig. 7).

\subsubsection{Morphological characterization of the membrane Ag/ Si SERS active substrates}

Figure 8a demonstrates the SEM image of the morphology of the fabricated silicon membrane with deposited nanoplasmonic silver nanoparticles inside the membrane channels.

To find out if the reduction of the silver ions on the HF etched silicon membrane took place and the porous silver nanoparticle aggregates in the silicon membrane channels were formed, the following experiment has been carried out. The pores of the blank membrane were characterized by the laser. The scheme of the measurement experiment is presented in Fig. 9. Before deposition of the silver nanoparticles, the laser light was visible through the membrane on the screen, as is seen from Fig. 10a. This means that the membrane was full of microchannels passing through the membrane.

The experiment was repeated after the deposition of the silver nanoparticles on the membrane. As seen from Fig. 10b, the laser light was still visible. However, the laser light intensity passing the membrane pores was lower and can be controlled by Ag deposition time. It means the silicon membrane channels are filled with Ag nanoparticles and produce an $\mathrm{Ag}$ nanoplasmonic filter type layer, working as a 3D tube type SERS channel with porous Ag nanoparticles film. 

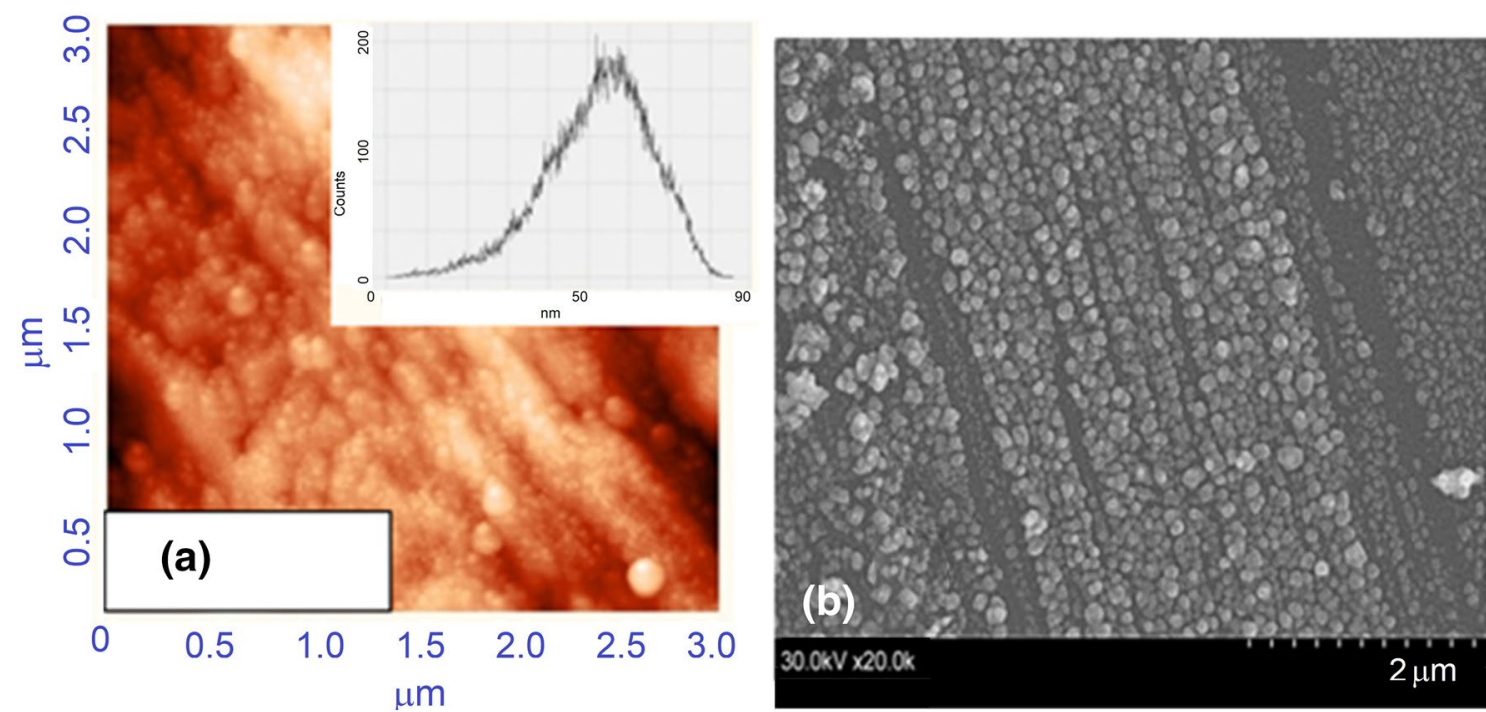

Fig. 6 Images of AFM scanning area of the silver SERS (a) and SEM image (b)
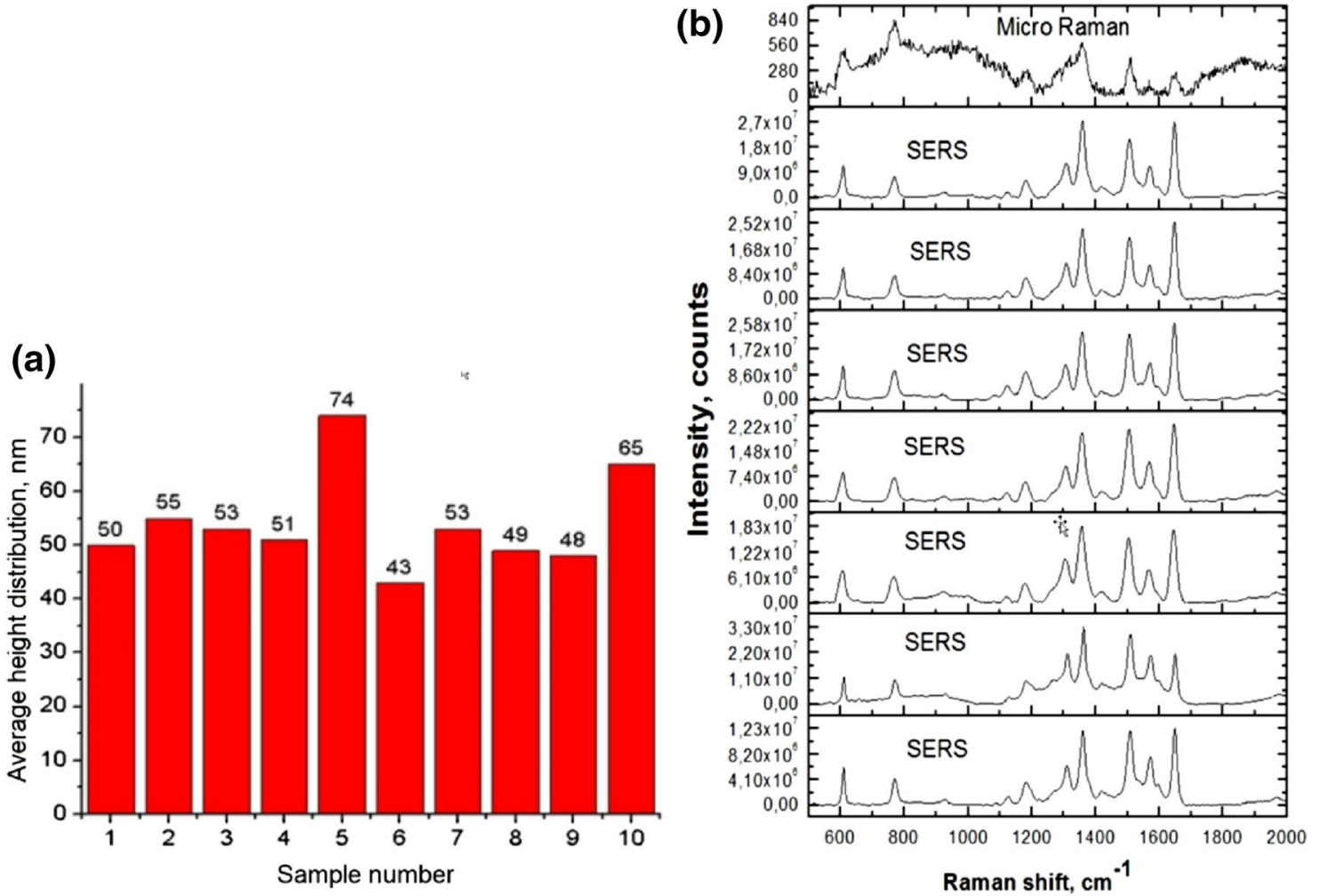

Fig. $7 \mathrm{Ag}$ /silicon SERS chip roughness repeatability (a), and SERS spectra of crystal violet solution at different spots (b)

The experiment revealed the deposition of the nanoparticles on the silicon membranes does not clog pores completely and the process can be controlled. Therefore, such a membrane integrated into the developed gas-pumping system would be able to pass the gaseous compounds through the pores full of plasmonic nanoparticles leading to the Raman signal enhancement. The focus spot diameter of the Raman laser beam used in our experimental setup was $500 \mathrm{~nm}$. The estimated number of spots with a high enhancement factor ("hot spots") can be dozens and the total counts of the SERS signal is a sum of scattered signals from the all excited "hot spots" in the volume of laser beam focus. As it is seen from 


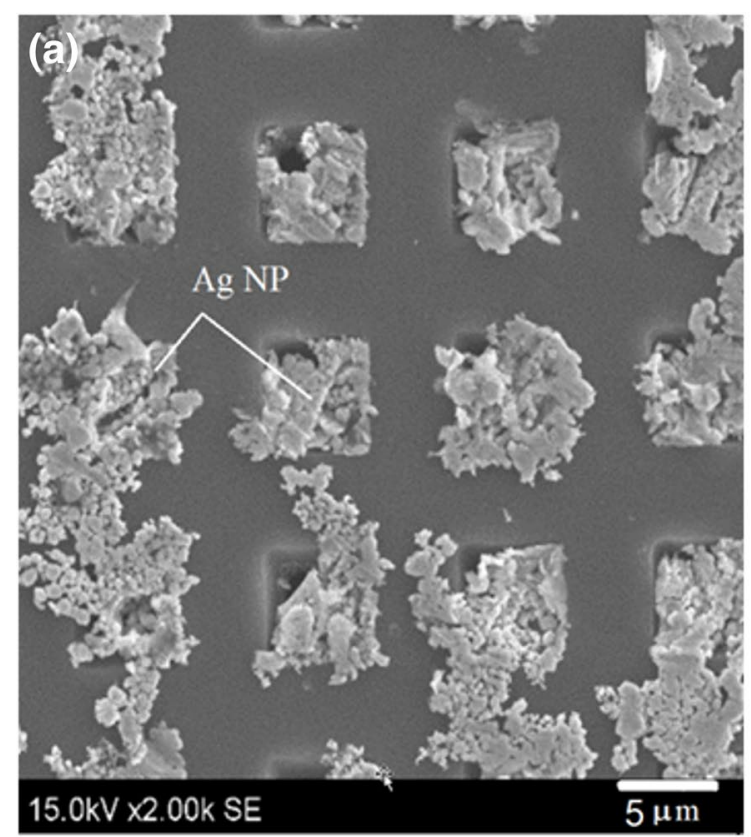

(b)

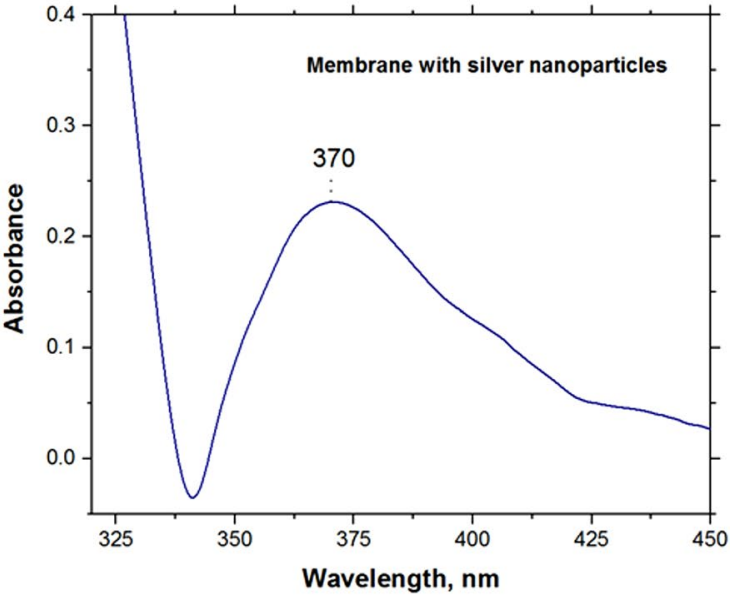

Fig. 8 SEM images of Si membranes decorated with silver nanoparticles (a) and UV-Vis spectra of the membrane with silver nanoparticles (b)

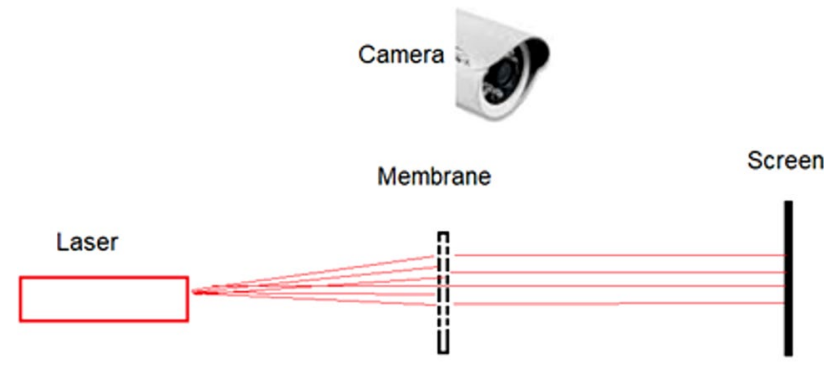

Fig. 8, the SERS substrate has densely packed with Ag nanoparticles aggregates in the pores and the probability for gas molecules pumped through the membrane and being trapped by "hot spots" increases. The molecules passing the plasmonic nanogap will be excited even if they are not adsorbed and the total Raman scattering signal and sensitivity of the sensor will be increased.

Fig. 9 Scheme of an experiment to test the Ag nanoparticles growth and to control the permeability of the membrane pores
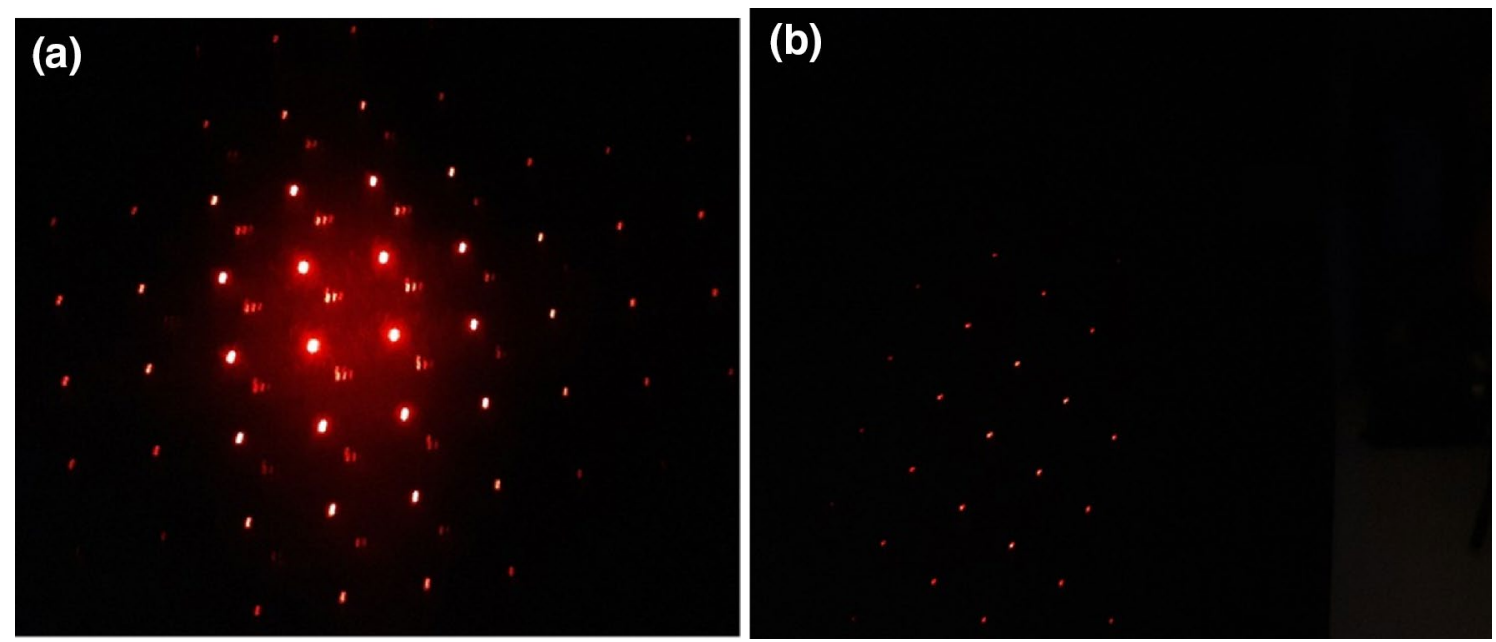

Fig. 10 Photos of the permeability of the laser light of the porous Si membrane: blank membrane with the pores of $5 \mu \mathrm{m}(\mathbf{a})$; membrane pores with silver nanoparticles (b) 


\subsection{Characterisation of SERS sensors for gas traces detection}

\subsubsection{Characterization of 3D Ag/Si SERS based sensors for gas trace detection}

The possibility to detect a trace concentration of gas molecules depends on SERS substrate metallic structure's capability to adsorb molecules from the environment. Many of VOC's molecules have a low adsorbtion coefficient in the gas phase. It depends on the polarity of gas molecules under investigation. The polar molecule of hydrazine and nonpolar anisole were selected for testing as members of the most frequently detected contaminants in spacecraft cabin [9]. The test measurements to characterize the manufactured sensor needs the known concentration of analyte gas volume in the container. The methodology to prepare the known concentration of the analyte in the container was the same as described in our previous work [23]. The detection of hydrazine from the aqueous solution as well as from the vapor phase using an analytical Raman platform was carried out. The solutions of hydrazine were prepared in distilled water. The concentrations of the hydrazine were: 100,50 , 25,10 , and $1 \mathrm{pM}$. The results of the hydrazine detection from the solutions are presented in Fig. 11. The assignments

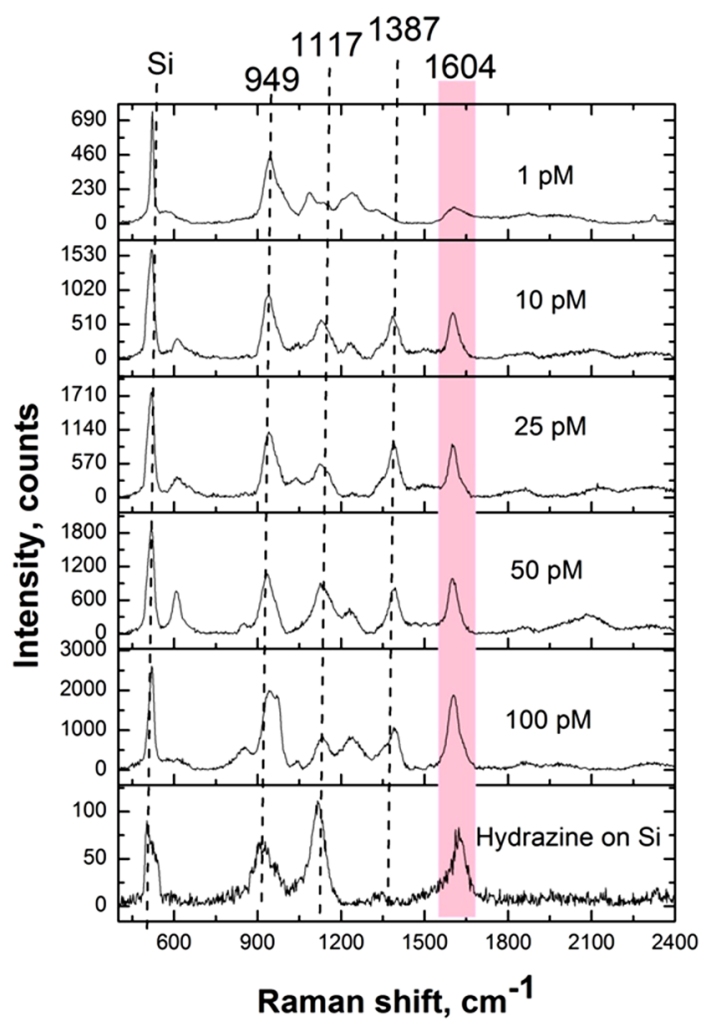

Fig. 11 SERS spectra of hydrazine detected from solution on $\mathrm{Ag} / \mathrm{Si}$ SERS substrate. Acquisition time: $20 \mathrm{~s}$ of the peaks that correspond to the vibrations of hydrazine are listed in Table 1.

Hydrazine was also detected from the vapor phase using an analytical Raman platform. The drop of concentrated hydrazine was dropped on the $\mathrm{Si}$ wafer. The sample was placed into the Petri dish. The 3D Ag/Si SERS substrate was placed in the same Petri dish as the Si wafer with the deposited concentrated hydrazine. The Petri dish was covered with the coverglass and left for $10 \mathrm{~min}$ to allow the hydrazine to evaporate completely and the SERS spectra measurements of hydrazine were performed. The results of the hydrazine detection from the vapor phase are presented in Fig. 12. As shown in Fig. 12, the main peaks corresponding to hydrazine were detected. It is important to notice that hydrazine from the vapor phase (as well as from solution) was detected on non-functionalized silver SERS substrates.

To test the possibility to measure trace concentration of non-polar gas molecules with 3D Ag/Si SERS substrate $100 \mu \mathrm{l}$ of liquid anisole was inserted into a $10 \mathrm{~L}$ volume container. The anisole evaporated and the final concentration of the gaseous anisole in the closed container was determined

Table 1 Assignments of hydrazine SERS peaks

\begin{tabular}{lc}
\hline Vibrational mode & Wavenumber $\mathrm{cm}^{-1}$ \\
\hline $\mathrm{NH}_{2}$ rocking & 590.5 \\
$\mathrm{~N}-\mathrm{N}$ stretching & 949.2 \\
$\mathrm{NH}_{2}$ wagging & 1115.8 \\
$\mathrm{NH}_{2}$ twisting & 1386.2 \\
$\mathrm{NH}_{2}$ scissor & 1603.6 \\
\hline
\end{tabular}

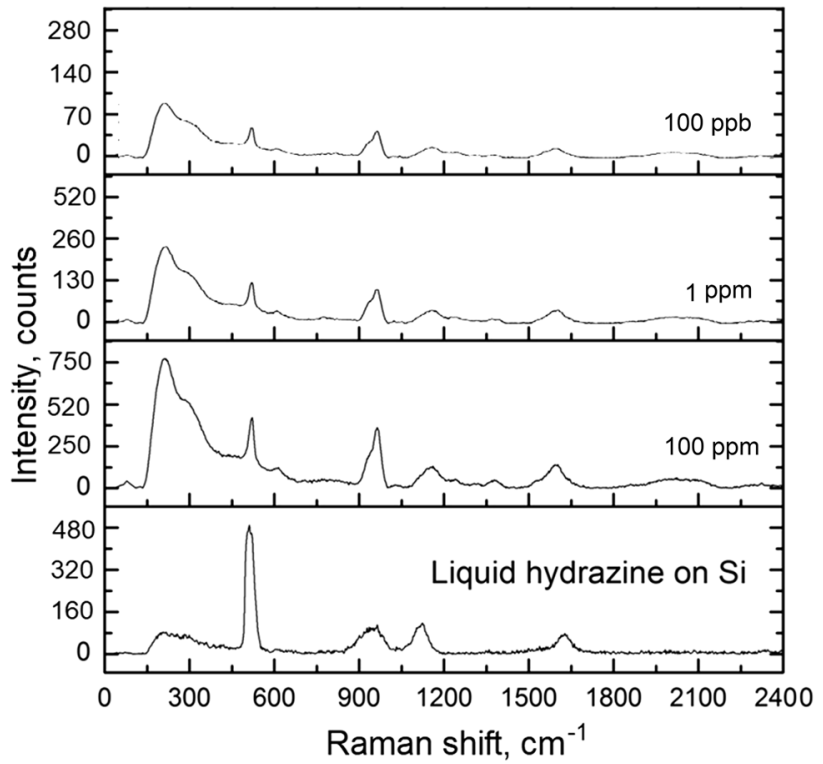

Fig.12 SERS spectra of hydrazine detected from the vapor phase on $\mathrm{Ag} / \mathrm{Si}$ SERS substrate. Acquisition time: $40 \mathrm{~s}$ 
$100 \mathrm{ppb}$ according to the methodology of the work [19]. The 3D Ag/Si SERS substrate was inserted into the closed vessel with anisole vapor for SERS spectra measurement to test the hypothesis of the anisole vapor adsorbtion on the SERS substrate. For this purpose, a sample of SERS was kept for $10 \mathrm{~min}$. in a closed vessel with anisole vapor, then removed and immediately placed under the objective of a Raman spectrometer, and the SERS spectrum recorded. As a result, the spectra of the anisole was at the level of measurement noise (Fig. 13).

\subsubsection{Characterization of Ag/Si membrane SERS based sensors for gas trace detection}

To ensure the reliability of the test measurement of the developed sensor, a clean environment was prepared for the preparation of the analyte gas volume. A clean glass vessel was prepared by washing it with ethanol and deionized water. The vessel was sealed with a flexible rubber membrane with sealed inlets for gas supply and exhaust. The vessel was purged of air by feeding high purity nitrogen gas to the vessel. A pressure of 0.5 bar was maintained at the inlet of the vessel and nitrogen gas was fed into the vessel for $10 \mathrm{~min}$ to completely expel air from the vessel. After purging the vessel of potentially molecularly contaminated air and filling it with clean nitrogen gas, a drop of liquid anisole was introduced into the vessel. The droplet size was chosen to produce the anisole vapor concentration required for the test measurement in the vessel. A drop of liquid anisole was introduced through the rubber membrane with the help of a syringe with a needle. After evaporation of the anisole drop, the vapor concentration in the vessel was calculated using a previously published methodology[19], which depends on the volume of liquid

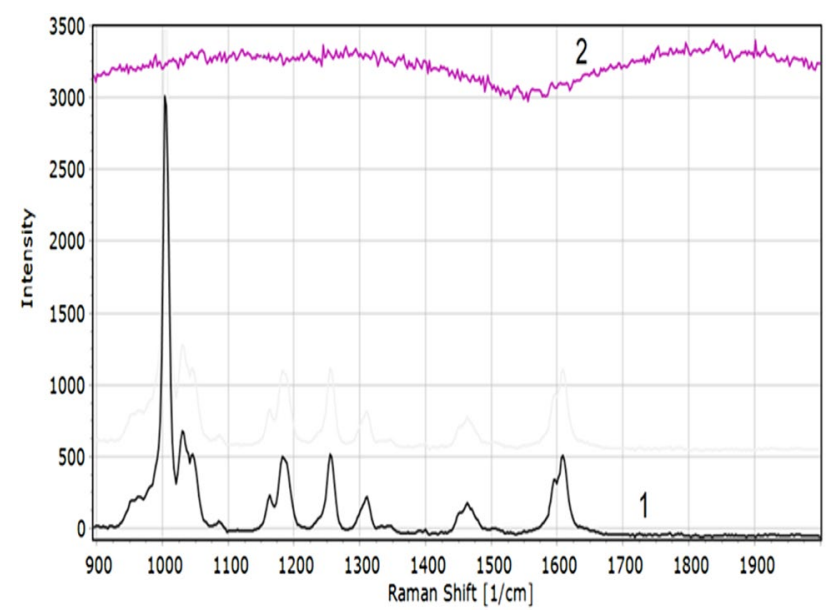

Fig.13 SERS spectrum of liquid anisole (1) and $100 \mathrm{ppb}$ concentration anisole (2) on $\mathrm{Ag} / \mathrm{Si}$ 3D substrate anisole drop introduced into the closed vessel. The anisole vapor in the air with the concentration of $10 \mathrm{ppmV}$ and 0.5 $\mathrm{ppbV}$ was selected for test measurement. After forming the volume of anisole vapor with the required concentration in the sealed vessel and removing the syringe, the anisole vapor is pumped into a molecular sensor with a SERS membrane through a connecting tube between the anisole vapor vessel and the gas micropump. The gas through the silver-coated membrane plasmon channels expels gas from the sensor chip. The gas delivery rate to the SERS sensor membrane was selected at $10 \mathrm{ml} \mathrm{min} \mathrm{m}^{-1}$ based on computer simulation studies of gas flow dynamics. The SERS signal was measured and recorded in real-time on a Raman spectrometer focusing the exciting laser beam on the surface of the SERS membrane. To ensure measurement reliability, the laser beam was scanned over a $20 \times 20$ micron membrane area during the measurement, integrating the SERS signal from multiple membrane channels filled with precipitated silver nanoparticles. The measurement platform schematic used for the test measurements is shown in Fig. 14a. Anisole vapor measurement results using an Ag/ Si membrane-type SERS sensor are presented in Fig.14b.

The results of the test measurements from the SERS spectrum shown in graph A. Figure 14 demonstrates that the membrane coated with silver nanoparticles do not have the characteristic peaks of organic pollutants in the nitrogen environment. It means the test environment in the vessel is free of unwanted molecules. The results of SERS test measurements in the environment with different anisole concentration and detected using the development platform, Fig. 14a, is presented in Fig. 14b. The spectra $\mathrm{B}$ and $\mathrm{C}$ demonstrate the results of anisole vapor with concentrations $10 \times 10^{-5} \mathrm{M}$ and $10 \times 10^{-9} \mathrm{M}$, respectively. The SERS spectra of gas-phase anisole demonstrate the same characteristic peaks as the spectra of liquid anisole; however, the intensity of the peaks around the shift of $1000 \mathrm{~cm}^{-1}$ is different. In the gas-phase anisole, the dominating peak is $1026 \mathrm{~cm}^{-1}$ and in the spectrum of liquid anisole, the dominating peak is $1005 \mathrm{~cm}^{-1}$. The spectrum of gas-phase anisole is shifted by $5 \mathrm{~cm}^{-1}$ down. It can be explained by the different excitation beam polarization effects during Raman spectra measurements and in the nanostructured SERS silver substrate. The presented measurement results of the developed hybrid membranetype SERS sensor demonstrate the extremely high sensitivity of organic gas molecules detection. The developed SERS-based molecular sensor can be applicable for contaminants detection in gas and liquid environment up to ppb concentrations with the possibility to recognize the type of molecule. The SERS molecular sensors open new directions in space research. 
Fig.14 Experimental setup of the anisole vapor pumping and Raman scattering measuring platform (a); The SERS spectra of the anisole after the background removal (b): blank Si membrane decorated with silver nanoparticles (A); anisole vapor with the concentration of $10 \mathrm{ppmV}$. The spectra acquisition time: $20 \mathrm{~s}$, the power of the laser on the sample: 0,17 $\mathrm{mW}$ (B); anisole vapor with the concentration of $0.5 \mathrm{ppbV}$. The spectra acquisition time: $60 \mathrm{~s}$, the power of the laser on the sample: $0,17 \mathrm{~mW}(\mathrm{C})$

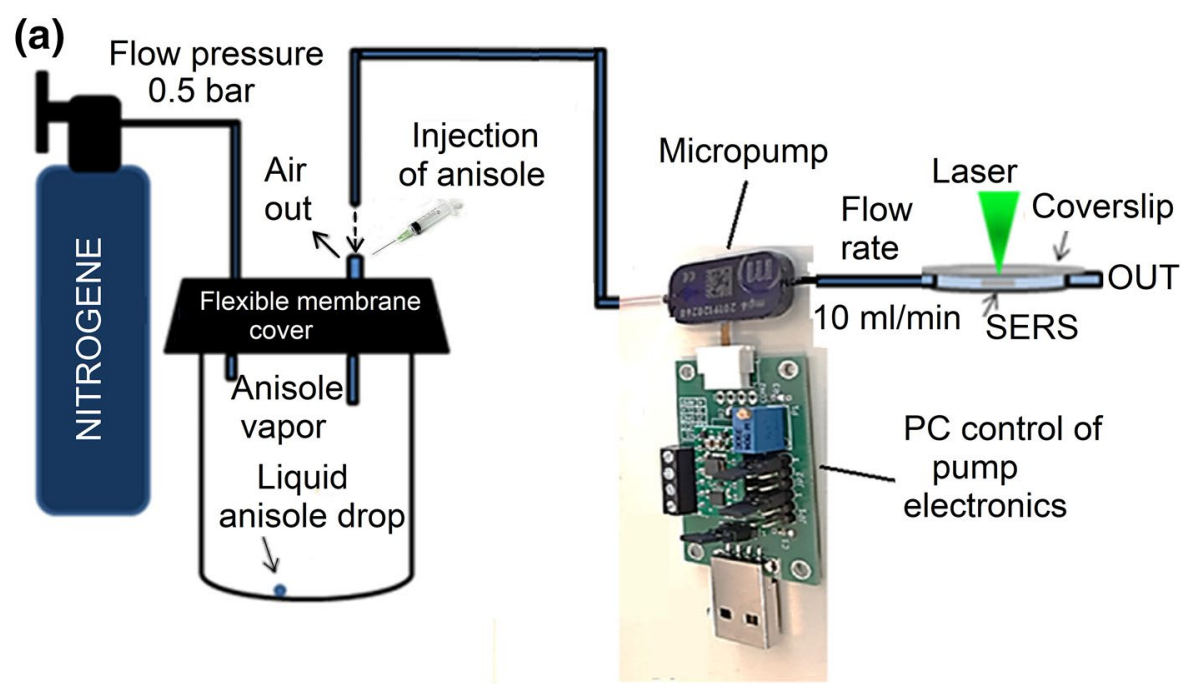

(b)

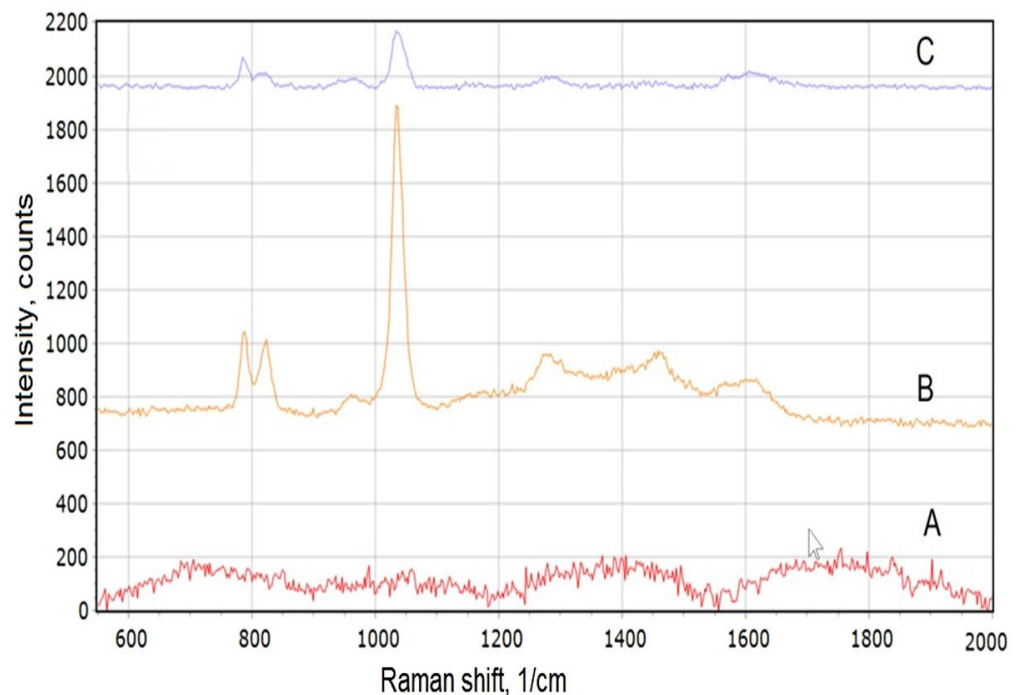

\subsection{Conclusions}

A novel silicon membrane covered with silver nanoparticles based SERS substrate has been developed. The SERS membrane molecular sensor provides high-sensitivity detection of VOC's in the gas and liquid environment. This research demonstrated the perspective concept of using the silicon micromachined membranes decorated with silver nanoparticles for sensitive detection of VOC's in the gas phase. Integration of membrane-based SERS sensors with micropumps and hand-held Raman spectrometers expands the applicability of this new technology in the areas of space engineering and research, environment contamination control, biomedical diagnostics, security purposes, and gas agents detection in the field. The measured hydrazine in solution molecular concentration using Ag SERS detection was of $1 \times 10^{-12} \mathrm{M}$ level that is $10^{3}$ order of magnitude lower compared to standard techniques. The detected hydrazine level in the gas-phase was at $0.1 \mathrm{ppmV}$ level. The measured nonpolar molecule anisole concentration in gas-phase using $\mathrm{Ag} / \mathrm{Si}$ membrane SERS with micropump was $0.5 \mathrm{ppbV}$. In summary, the results demonstrated by this work open new ways for the development of the next-generation molecular sensing systems with the possibility to detect ultralow concentrations of VOC's in various surroundings, which is not achievable by standards techniques. The Raman spectra-based fingerprinting makes it possible not only to detect the molecules but at the same time to recognize the molecule's type. The testing of this technique to detect molecular contamination in liquids is the next step of our research.

Supplementary Information The online version contains supplementary material available at https://doi.org/10.1007/s12567-021-00356-6.

Acknowledgements This work was funded through a European Space Agency Contract 4000126337/18/NL/SC under the PECS (Plan for the European Cooperating States). The view expressed herein can in 
no way be taken to reflect the official opinion of the European Space Agency. The authors would like to thank Dr. Lina Traksele for her help in providing SERS measurements and Kestutis Nemciauskas for computer simulations.

Author contributions VS, UL, YB, CS contributed to the study conception and design. Material preparation, data collection, and analysis were performed by VS, DB, IB, UL, YB. The first draft of the manuscript was written by VS and all authors commented on previous versions of the manuscript. All authors read and approved the final manuscript.

\section{Compliance with ethical standard}

Conflict of interest There are no competing interests that are directly or indirectly related to the work submitted for publication. The authors have no financial or proprietary interests in any material discussed in this article.

\section{References}

1. Contamination Control Handbook, Prepared by Sandia Laboratories, NASA SP-5076. Office of Technology Utilization, National Aeronautics and Space Administration, 1969

2. Kesselmeier, J., Staudt, M.: Biogenic volatile organic compounds (VOC): an overview on emission, physiology and ecology. J. Atmos. Chem. 33, 23-88 (1999)

3. Galiana, H.L.: University role in astronaut life-supporting systems: monitoring atmospheric contaminants. Massachusets Institute of Technology, NASA CR-1826, 1971

4. Madzunkov, S. M., Bae, B., Simcic, J., Rellergert, W., Gill, J., Schaefer, R., Neidholdt, E., Homer, M. L., Nikolić D., Kidd R.D., Darrach M.: Progress report on the spacecraft atmosphere monitor. Proceedings of 46th International Conference on Environmental Systems ICES-2016-284, 10-14 July 2016, Vienna, Austria, paper ICES-2016-284

5. Young, R.C., Buttner, W.J., Linnell, B.R.: Rajeshuni Ramesham Electronic nose for space program applications. Sensors Actuators B 93, 7-16 (2003)

6. Walper, S.A., Aragonés, G.L., Sapsford, K.E., Brown, C.W., Breger, J.C., Medintz, I.L.: Detecting biothreat agents: from current diagnostics to developing sensor technologies. ACS Sens 3, 1894-2024 (2018)

7. Peterson, V.B., et al.: Characterization of microbial and chemical composition of shuttle wet waste with permanent gas and volatile organic compound analyses. Adv. Space Res. 34, 1470-1476 (2004)

8. Romoser, A.A., et al.: Predicting air quality at first ingress into vehicles visiting the International Space Station. Aerospace Med. Hum Perform. 88(2), 104-113 (2017)

9. Perry, J.L.: Trace Contaminant Control for the International Space Station's Node 1-analysis, design, and verification. National
Aeronautics and Space Administration, Marshall Space Flight Center, NASA/TP-2017-218235 (2017)

10. Calaway, M.J., Allen, C.C., J.H. Allton Organic Contamination Baseline Study in NASA Johnson Space Center Astromaterials Curation Laboratories, NASA TP-2014-217393, pp. 1-69, 1 July 2014

11. Wallace, W.T., Gazda, D.B., Limero, T.F., Minton, J.M., Macatangay, A.V., Dwivedi, P., Fernandez, F.M.: Electrothermal vaporization sample introduction for spaceflight water quality monitoring via gas chromatography-differential mobility spectrometry. Anal. Chem. 87, 5981-5988 (2015)

12. Limero, T.F., Trowbridge, J.B., Wallace W.T. In: 43rd International Conference on Environmental Systems, p. 3484, AIAA, Vail, CO, 2013

13. McCoy, J.T., Flint, S., Straub, J., Gazda, D.B., Schultz J. In: 41st International Conference on Environmental Systems, p. 5153, AIAA, Portland, OR, USA, 2011

14. Yuan, Z., Li, R., Meng, F., Zhang, J., Zuo, K., Han, E.: Approaches to enhancing gas sensing properties: a review. Sensors (Basel) 7 , 1495 (2019)

15. Qian, X., Nie, S.: Single-molecule and single-nanoparticle SERS: from fundamental mechanisms to biomedical applications. Chem. Soc. Rev. 37, 912-920 (2008)

16. Singh, E., Meyyappan, M.: Flexible graphene-based wearable gas and chemical sensors. ACS Appl. Mater. Interfaces 9, 34544 34586 (2017)

17. European Network on new sensing technologies for air-pollution control and environmental sustainability (EuNetAir). http://www. cost.eunetair.it/. Accessed 3 Jan 2021

18. Samsung SARS-CoV-2 detection with SERS spectroscopy research, https://devpost.com/software/samsung-research-forsars-cov-2-detection-with-spectroscopy, (accessed 03.01.2021).

19. Ramanauskaite, L., Snitka, V.: The synthesis of controlled shape nanoplasmonic silver-silica structures by combining sol-gel technique and direct silver reduction. Nanoscale Res. Lett. 10, 133 (2015)

20. Rae, S., Khan, I.: Surface enhanced Raman spectroscopy (SERS) sensors for gas analysis. Analyst 135(6), 1365-1369 (2010)

21. Sweetenham, C.S., Notingher, I.: Combined atomic force microscopy-Raman mapping of electric field enhancement and surfaceenhanced Raman scattering hotspots for nanosphere lithography substrates. J. Nanophotonics 5, 059504-059504 (2011)

22. https://www.effemm2.de/spectragryph/. Accessed 3 Jan 2021

23. Ramanauskaite, L., Mazeika, V., Snitka, V.: SERS based monitoring of toluene vapors at ambient and elevated temperatures by using a ruffled silver nanolayer as a substrate. Microchim. Acta 185, 477 (2018)

Publisher's Note Springer Nature remains neutral with regard to jurisdictional claims in published maps and institutional affiliations.

\section{Authors and Affiliations}

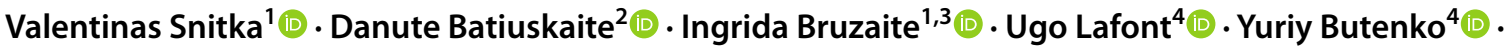 Christopher Semprimoschnig ${ }^{4}$}

Danute Batiuskaite

danute.batiuskaite@vdu.lt
Ingrida Bruzaite

ingrida.bruzaite@vgtu.lt 
Ugo Lafont

Ugo.Lafont@esa.int

Yuriy Butenko

Yuriy.Butenko@esa.int

1 Research Center for Microsystems and Nanotechnology, Kaunas University of Technology, Studentu 65, Kaunas, Lithuania

2 Department of Biology, Faculty of Natural Sciences, Vytautas Magnus University, 58 K.Donelaicio str., 44248 Kaunas, Lithuania
3 Department of Chemistry and Bioengineering, Faculty of Fundamental Sciences, Vilnius Gediminas Technical University, 10223 Vilnius, Lithuania

4 European Space Research and Technology Centre (ESTEC), European Space Agency (ESA), Keplerlaan 1, Postbus 299, 2200 AG Noordwijk, The Netherlands 